\title{
Desarrollo de plan de entrenamiento con simulación de artroscopía para residentes de ortopedia
}

\author{
Arroyo-Berezowsky C* \\ Centro Médico ABC
}

RESUMEN. Introducción: Cada vez hay menos oportunidades para que los residentes aprendan y desarrollen habilidades quirúrgicas en la sala de operaciones. En algunos lugares del mundo aparece el desarrollo y adaptación de la tecnología para la enseñanza de habilidades sicomotoras en las especialidades quirúrgicas. La artroscopía es una técnica quirúrgica que se presta a la simulación. Hasta ahora no existe en México un programa de entrenamiento estructurado y validado de artroscopía para residentes de ortopedia. Objetivo: Proponer un plan de entrenamiento de artroscopía estructurado que incorpore la simulación para residentes de ortopedia. Método: Se realizó una revisión de la literatura sobre enseñanza con simulación en ortopedia y artroscopía, se realizó un estudio para establecer valores de referencia para ejercicios de artroscopía en un simulador de realidad virtual y se desarrolló un plan de entrenamiento con simulación de artroscopía para residentes de ortopedia. Resultados: Se elaboró un programa de siete etapas con clases teóricas y simulación para mejoría de habilidades motoras. La complejidad de los procedimientos aumenta a través de los módulos prácticos. Se complementa con una evaluación semestral. Discusión: Actualmente la mayoría de los esfuerzos en enseñanza con simuladores se enfoca en estudiantes de medicina y residentes de primer y segundo año. Considero que se deben enfocar los esfuerzos para incluir residentes de mayores jerarquías y cirujanos. Se propondrá
ABSTRACT. Background: There are less and fewer opportunities for residents to learn and develop surgical skills in the operating room. In some parts of the world, the development and adaptation of technology for the teaching of psychomotor skills in surgical specialties appears. Arthroscopy is a surgical technique that lends itself to simulation. So far there is no such thing as a structured and validated arthroscopy training program for orthopaedic residents in Mexico. Objective: To propose a structured arthroscopy training plan that incorporates the simulation for orthopaedic residents. Method: A review of the literature on teaching with simulation in orthopaedics and arthroscopy was performed, a study was conducted to establish reference values for arthroscopy exercises in a virtual reality simulator and a training plan was developed with Arthroscopy simulation for orthopaedic residents. Results: A seven-stage program was developed with theoretical classes and simulation to improve motor skills. The complexity of the procedures increases through the practical modules. It is complemented by a semester evaluation. Discussion: Currently most of the efforts in teaching with simulators focus on medical students and first-and secondyear residents. I believe that efforts should be focused to include residents of higher ranks and surgeons. This design will be proposed to the orthopaedic Unique Medical Education Program (PUEM in Spanish). It is important to develop

* Ortopedista del Centro Médico ABC.

Dirección para correspondencia:

Dra. Claudia Arroyo Berezowsky

Centro Médico ABC

Consultorio 1 Módulo C Torre Sur, Sur 136 Núm. 116, Col. Las Américas, CP 01120, Álvaro Obregón, CDMX.

E-mail: dra.carroyob@gmail.com 
incluir este diseño al Programa Único de Educación Médica (PUEM) de ortopedia. Es importante desarrollar planes de estudio validados que incorporen la simulación para el desarrollo de habilidades motoras en residentes de ortopedia.

Palabras clave: Simulación, artroscopía, residente, enseñanza, realidad virtual.

\section{Introducción}

La enseñanza médica experimentó cambios de paradigmas en las últimas décadas, principalmente la enseñanza de habilidades quirúrgicas. ${ }^{1,2}$ Con los cambios en los programas de residencia médica en Estados Unidos, como restricciones en los horarios de trabajo, mayor riesgo de demandas médico-legales, mayor costo del quirófano y mayor dificultad técnica de algunos procedimientos quirúrgicos, hay menos oportunidad para que los residentes aprendan y desarrollen habilidades quirúrgicas en la sala de operaciones. ${ }^{1,2,3,4}$ Asimismo, existe la necesidad de documentar la adquisición de estas habilidades de los residentes de manera objetiva. ${ }^{5,6}$ Estos cambios han impulsado el desarrollo y adaptación de la tecnología para la enseñanza de habilidades sicomotoras en las especialidades quirúrgicas. También se ha buscado el desarrollo e incorporación de programas de entrenamiento con simulación al currículo de las residencias de ortopedia. ${ }^{1,5,6,7}$

Algunas de las propuestas han sido dividir el aprendizaje de un procedimiento quirúrgico en la práctica y dominación de habilidades básicas individuales para permitir que el residente tenga un mejor desempeño global. ${ }^{5,6,8,9}$ Merrinböer expresó que una cirugía no sólo es la suma de varias habilidades individuales, también incluye la habilidad para coordinar e integrar esas partes y saber reaccionar ante dificultades no previstas. ${ }^{10}$

La cirugía artroscópica es un procedimiento quirúrgico demandante técnicamente y constituye uno de los procedimientos ortopédicos más comunes en la actualidad. Requiere coordinación visoespacial para manipular instrumentos mientras se interpretan estructuras en tercera dimensión en una imagen en dos dimensiones..$^{11} \mathrm{La}$ artroscopía se presta para la simulación. Existen varios tipos de simuladores en artroscopía: simuladores cadavéricos, maniquíes y simuladores de realidad virtual. ${ }^{12}$

Estudios han demostrado mejoría de habilidades artroscópicas en los residentes después de recibir entrenamiento con cualquier tipo de simulador. ${ }^{13,14,15,16}$ Sin embargo, se reconoce que actualmente hay poca consistencia en el uso de simuladores en enseñanza. ${ }^{3,17}$ También se ha demostrado que tienen una buena capacidad de discriminación entre niveles de habilidad quirúrgica; aunque por el momento no se ha logrado documentar la transferibilidad de las habilidades del simulador al quirófano. ${ }^{18,19,20,21}$ Para evaluar la transferibilidad se han validated curricula that incorporate the simulation for the development of motor skills in orthopaedic residents.

Key words: Simulation, arthroscopy, resident, education, virtual reality.

utilizado escalas de evaluación global como: evaluación objetiva estructurada de habilidades quirúrgicas conocida como OSATS por sus siglas en inglés, la herramienta de evaluación de habilidades en cirugía artroscópica (Tabla 1) (ASSET por sus siglas en inglés), la escala imperial global de calificación artroscópica (IGARS por sus siglas en inglés) y el sistema básico de habilidades para artroscopía de rodilla (BAKSS por sus siglas en inglés). . $22,23,24,25,26,27$

Un programa de simulación debe ser progresivo, en el que estén bien definidas las habilidades básicas que se desean desarrollar. ${ }^{2}$ Hodgins y Unalan reportaron que los sujetos en proceso de desarrollo de nuevas habilidades tienen mejor desempeño si reciben retroalimentación verbal en tiempo real que los que no la reciben. ${ }^{11,13}$ También se debe contar con un sistema de evaluación objetivo para la mejoría de las habilidades artroscópicas. Los simuladores de realidad virtual reportan calificaciones al completar cada ejercicio y para el desempeño en quirófano se utilizan las escalas de validez de evaluación quirúrgica. El objetivo es desarrollar un programa de entrenamiento de artroscopía a cuatro años para residentes de ortopedia.

\section{Método}

Se realizó una revisión de la literatura disponible para enseñanza y simulación en ortopedia en PubMed y Google Scholar utilizando los términos MeSH: simulation training, orthopedics, resident, arthroscopy, virtual reality. Se incluyeron todos los artículos en los que se analizaron planes y problemas de enseñanza en ortopedia, los que evaluaron un plan de enseñanza con simuladores, aquéllos en los que se validó la mejoría de habilidades motoras de residentes con o sin simulación de artroscopía y aquéllos que validaron escalas de evaluación de habilidades motoras en quirófano. Se revisaron 11 artículos sobre el desarrollo de habilidades con simulación, siete sobre validación de las habilidades adquiridas, cuatro sobre enseñanza en ortopedia y cinco sobre escalas de evaluación en artroscopía y simulación.

Inicialmente se realizó un programa piloto en el que participaron médicos residentes de ortopedia y ortopedistas. En éste, los participantes realizaron una serie de ejercicios de artroscopía diagnóstica de rodilla en un simulador de realidad virtual. Los promedios para cada variable se establecieron como los valores de referencia para el futuro. En 


\begin{tabular}{|c|c|c|c|c|c|}
\hline \multicolumn{6}{|c|}{$\begin{array}{c}\text { Tabla 1: Escala de evaluación global ASSET. } \\
\text { Traducida de Koehler } 4\end{array}$} \\
\hline \multirow[t]{2}{*}{ Seguridad } & 1 - Novato & 2 & 3 - Competente & 4 & 5 - Experto \\
\hline & $\begin{array}{l}\text { Sobre tentativo o torpe con los } \\
\text { instrumentos, no puede dirigirlos } \\
\text { consistentemente a sus objetivos }\end{array}$ & & $\begin{array}{l}\text { Cuidadoso, uso controlado de los } \\
\text { instrumentos, ocasionalmente no } \\
\text { le atina a sus objetivos }\end{array}$ & & $\begin{array}{l}\text { Seguro de sí mismo, uso preciso } \\
\text { de todos los instrumentos }\end{array}$ \\
\hline \multirow[t]{2}{*}{ Campo de visión } & 1 - Novato & 2 & 3 - Competente & 4 & 5 - Experto \\
\hline & $\begin{array}{l}\text { Campo de visión angosto, } \\
\text { posición no adecuada de } \\
\text { artroscopio o fuente de luz }\end{array}$ & & $\begin{array}{c}\text { Campo de visión moderado, } \\
\text { adecuada posición de artroscopio } \\
\text { y fuente de luz }\end{array}$ & & $\begin{array}{c}\text { Campo de visión extenso, } \\
\text { posición óptima de artroscopio y } \\
\text { fuente de luz }\end{array}$ \\
\hline \multirow[t]{2}{*}{ Destreza con cámara } & 1 - Novato & 2 & 3 - Competente & 4 & 5 - Experto \\
\hline & $\begin{array}{l}\text { Movimientos torpes o sin gracia, } \\
\text { incapaz de mantener cámara } \\
\text { centrada y bien orientada }\end{array}$ & & $\begin{array}{c}\text { Adecuado uso de la cámara, } \\
\text { ocasionalmente requiere } \\
\text { recolocación }\end{array}$ & & $\begin{array}{l}\text { Movimientos diestros y gráciles } \\
\text { a lo largo del procedimiento con } \\
\text { la cámara siempre centrada y } \\
\text { correctamente orientada }\end{array}$ \\
\hline \multirow[t]{2}{*}{ Destreza bimanual } & 1 - Novato & 2 & 3 - Competente & 4 & 5 - Experto \\
\hline & $\begin{array}{c}\text { Incapaz de utilizar ambas manos } \\
\text { o sin coordinación entre ambas } \\
\text { manos }\end{array}$ & & $\begin{array}{c}\text { Utiliza ambas manos, } \\
\text { pero ocasionalmente poca } \\
\text { coordinación entre el movimiento } \\
\text { de cámara e instrumentos }\end{array}$ & & $\begin{array}{c}\text { Utiliza ambas manos para } \\
\text { coordinar cámara e instrumentos } \\
\text { y posicionarlos para un adecuado } \\
\text { desempeño }\end{array}$ \\
\hline \multirow[t]{2}{*}{ Flujo del procedimiento } & 1 - Novato & 2 & 3 - Competente & 4 & 5 - Experto \\
\hline & $\begin{array}{l}\text { Detiene el procedimiento } \\
\text { frecuentemente o persiste sin } \\
\text { progresar, intentos múltiples } \\
\text { fallidos antes de completar el } \\
\text { ejercicio }\end{array}$ & & $\begin{array}{l}\text { Progresión estable del } \\
\text { procedimiento quirúrgico con } \\
\text { pocos intentos fallidos antes de } \\
\text { completar el ejercicio }\end{array}$ & & $\begin{array}{l}\text { Orden del procedimiento } \\
\text { obviamente planeado, } \\
\text { transiciones fluidas de uno a otro } \\
\text { ejercicio sin intentos fallidos }\end{array}$ \\
\hline \multirow[t]{2}{*}{ Calidad de procedimiento } & 1 - Novato & 2 & 3 - Competente & 4 & 5 - Experto \\
\hline & $\begin{array}{l}\text { Producto final inadecuado o } \\
\text { incompleto }\end{array}$ & & $\begin{array}{c}\text { Producto final adecuado con } \\
\text { pequeñas fallas que no requieren } \\
\text { corrección }\end{array}$ & & Producto final óptimo sin fallas \\
\hline \multirow[t]{2}{*}{ Autonomía } & 1 & & 2 & & 3 \\
\hline & $\begin{array}{c}\text { Incapaz de completar } \\
\text { procedimiento incluso con } \\
\text { intervenciones }\end{array}$ & & $\begin{array}{l}\text { Capaz de completar } \\
\text { procedimiento pero requirió } \\
\text { intervenciones }\end{array}$ & & $\begin{array}{c}\text { Capaz de completar } \\
\text { procedimiento sin intervenciones }\end{array}$ \\
\hline Procedimiento de & 1 & & 2 & & 3 \\
\hline complejidad agregado & No dificultad & & $\begin{array}{l}\text { Dificultad moderada (inflamación } \\
\text { leve o fibrosis) }\end{array}$ & & $\begin{array}{c}\text { Dificultad extrema (inflamación } \\
\text { severa, anatomía anormal o } \\
\text { abundante fibrosis) }\end{array}$ \\
\hline
\end{tabular}

este estudio se encontraron diferencias que favorecieron a los médicos tratantes durante los primeros ejercicios. Pero después de una serie de ejercicios durante aproximadamente dos horas, no se encontraron diferencias estadísticamente significativas en el desempeño entre los médicos residentes y los médicos tratantes. Con esta información se elaboró un plan de entrenamiento de artroscopía para residentes de ortopedia incorporando la simulación.

\section{Resultados}

El plan de entrenamiento en artroscopía para residentes será progresivo en dificultad teórica y práctica durante los cuatro años de residencia. Como métodos didácticos auxiliares se utilizarán simuladores de caja, de realidad virtual de retroalimentación háptica, modelos en seco y cadavéricos.

El programa progresará a través de diferentes bloques con el objetivo de que cada residente desarrolle habilidades motoras independientes que pueda integrar a una artroscopía real. Estos bloques son: habilidades motoras básicas (como triangulación y navegación), navegación intraarticular, diagnóstico y tratamiento. Cada bloque de aprendizaje se compondrá de un ciclo de siete etapas. Estas siete etapas incorporan clases teóricas, simulación y evaluación de artroscopías reales (Figura 1). La primera etapa consta de sesiones de 10 minutos enfocadas en la realización de cada ejercicio. La segunda etapa es una demostración del ejercicio a realizar en el simulador que corresponda por parte del instructor. La tercera etapa incluye la simulación. Los residentes de primer año inician su entrenamiento en simuladores básicos. Son cajas con nueve orificios en la superficie superior a través de los que se pueden introducir una cámara e instrumentos. En el interior de la caja se pueden colocar diferentes objetos que se deben manipular para aprender a navegar en dos dimensiones. Cuando dominen los ejercicios disponibles con ambas manos, pueden progresar al simulador de realidad virtual, éste es de retroalimentación háptica. El usuario experimenta resistencia a través de los instrumentos si se manipulan estructuras dentro de la articulación. Cuenta con un currículo académico preinstalado basado en uno creado por la Asociación Norteamericana de Artroscopía (AANA por sus siglas en inglés) que se utiliza durante los cursos de artroscopía. 


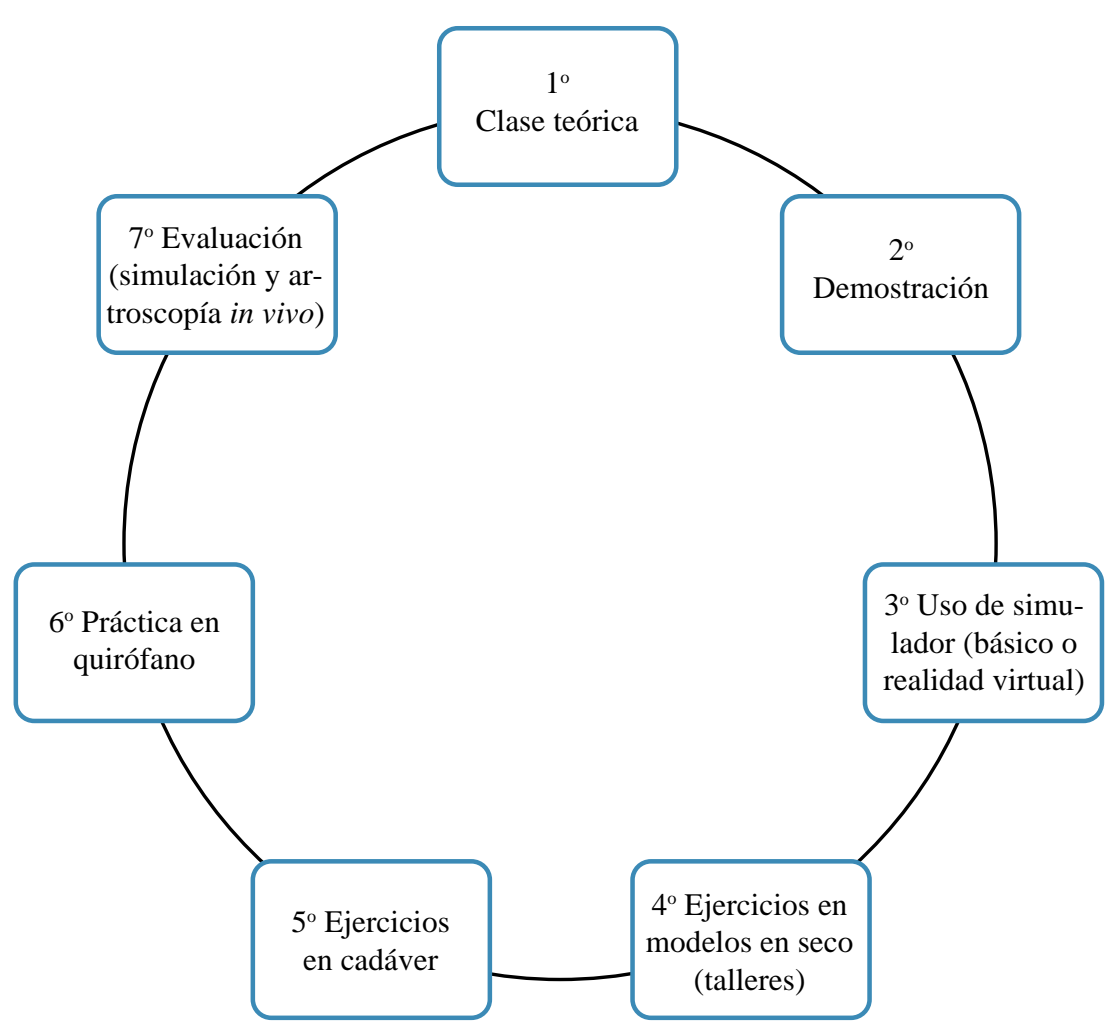

Figura 1:

Las siete etapas del plan de entrenamiento por simulación para residentes de artroscopía.

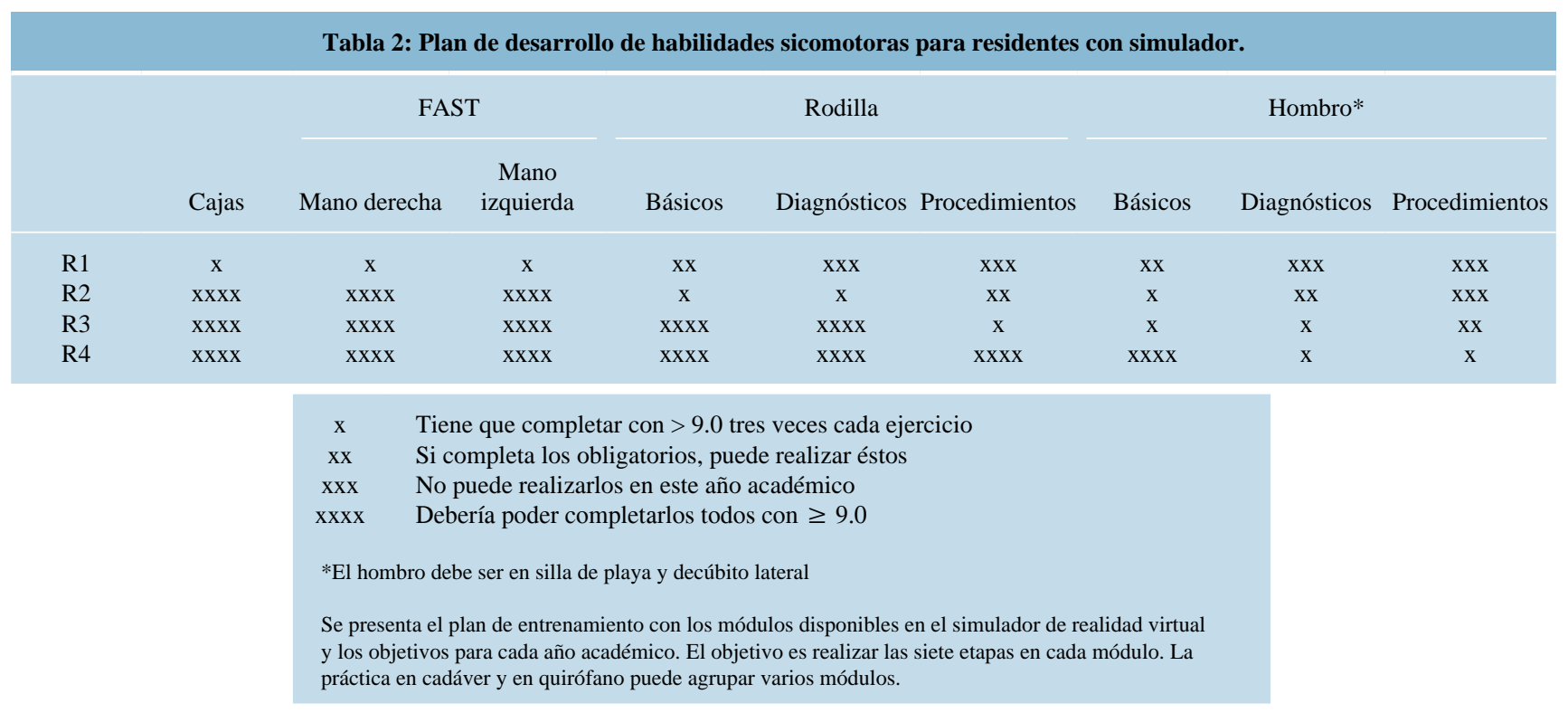

Los residentes podrán progresar a través de los módulos del currículo virtual de acuerdo con su grado académico (Tabla 2). Hay módulos que deberán completar de manera obligatoria cada año. Tendrán la posibilidad de progresar al siguiente bloque si cumplen con los requerimientos establecidos. Habrá otros bloques a los que no podrán avanzar sin importar su desempeño, ya que estarán destinados para los siguientes grados académicos.

Los residentes deben lograr realizar cada ejercicio tres veces con una calificación global mayor o igual a nueve. En caso de no cumplir con estos requisitos, no podrán progresar al siguiente año académico.

En la cuarta etapa se realizarán talleres en modelos en seco. La quinta etapa incluye prácticas en cadáver para procedimientos diagnósticos y terapéuticos. En la sexta etapa podrá participar más activamente en las cirugías de artroscopía.

La séptima etapa corresponde a la evaluación. Al principio del año académico los residentes de nuevo ingreso deberán realizar una secuencia de ejercicios básicos y ar- 
troscopía diagnóstica de rodilla con y sin gancho palpador con recorrido fijo. De manera semestral, todos los residentes deberán realizar una artroscopía diagnóstica en el simulador de realidad virtual. Los de primer y segundo año de rodilla y tercer y cuarto de rodilla y de hombro. De esta manera se contará con valores de referencia para documentar mejoría.

Además, el residente deberá realizar una artroscopía diagnóstica en rodilla o en hombro en quirófano, bajo la supervisión de un médico adscrito y deberá recabar el video de la cirugía. Este video será calificado por el instructor de simulación de manera independiente y anónima utilizando la escala de evaluación global de ASSET que ha sido validada para evaluar el desempeño en una artroscopía.

\section{Discusión}

En la sexta junta anual del consorcio de institutos acreditados para la educación del Colegio Americano de Cirujanos (ACS-AEI por sus siglas en inglés) se concluyó que es necesario un esfuerzo interdisciplinario para compartir avances en simulación en todas las especialidades quirúrgicas. Actualmente, la mayoría de los esfuerzos de enseñanza con simuladores se enfocan en estudiantes de pregrado y residentes de primer y segundo año. Se debe incluir a los residentes de mayores grados académicos, cirujanos que deban aprender nuevas habilidades, cirujanos que deban mantener habilidades quirúrgicas y tal vez utilizarlos como método de evaluación para certificación o recertificación. Los cirujanos retirados o cercanos a la edad de retiro podrían ser reclutados como instructores y profesores para a los residentes. ${ }^{14}$

Se cree que la principal diferencia entre cirujanos expertos y novatos en artroscopía recae en que los primeros tienen más experiencia en la ponderación sensorial de señales distractoras y no necesariamente mayor experiencia en la manipulación de instrumentos y tejidos. ${ }^{10,13}$ Para poder realizar una cirugía completa, un cirujano requiere un gran repertorio de habilidades motoras básicas además de la capacidad de adaptarse a situaciones desconocidas, reaccionar ante ellas y ser capaz de integrar todos los estímulos sensoriales que recibe dentro y fuera del campo quirúrgico. ${ }^{10,13}$

Hasta ahora, los simuladores de baja y de alta fidelidad han demostrado valor de enseñanza en diversas especialidades quirúrgicas, se ha demostrado que tienen una buena validez interna, pero no se ha logrado validar la transferibilidad de habilidades del simulador al quirófano (validez externa). ${ }^{18,19,20,21,22,23,24,25,26,27}$ Idealmente deben incorporarse distractores a la simulación ya que se dominan las habilidades básicas. El entrenamiento debe complementarse con una evaluación en cirugía real con una escala validada para documentar mejoría global y la transición de cirujano novato a cirujano experto. ${ }^{10,11,12,13}$

Uno de los principales problemas con la estructuración de los programas de enseñanza con simulación y la validación de los simuladores es que no se ha prestando suficiente atención a la necesidad de modelos de entrenamiento basados en competencias que puedan ayudar a los residentes a alcanzar suficiencia en habilidades específicas y que, a la vez, puedan reafirmar habilidades en estos niveles. ${ }^{14}$ Se ha demostrado que la práctica y entrenamiento continuos mantienen una habilidad por un período de hasta tres años. También se ha demostrado que si un programa de entrenamiento es seguido de una sesión de práctica, se reforzará lo aprendido. ${ }^{10}$ Actualmente todos los esfuerzos de investigación se están concentrando en resolver estos dilemas para poder diseñar programas de entrenamiento formales y homogéneos, que puedan ser aplicados globalmente. ${ }^{14}$

El plan de entrenamiento propuesto aquí puede ser incorporado al Programa Único de Educación Médica (PUEM) de ortopedia. El objetivo es organizar y estructurar el aprendizaje teórico, la participación en talleres y en quirófano en los que ya participa la mayoría de las sedes hospitalarias, así como complementar la práctica de los residentes y proveer un espacio en el que puedan cometer errores sin comprometer la seguridad del paciente.

En estudios previos para validar el simulador se encontró que la artroscopía diagnóstica y otros ejercicios, así como el número de colisiones, tiempo para completar el ejercicio y distancia viajada por los instrumentos pueden discriminar entre niveles de habilidad quirúrgica. ${ }^{18,19,20,21}$ Sin embargo, no existen en la literatura internacional valores meta para estas variables o una calificación determinada que relacione el desempeño en el simulador con el desempeño en una cirugía real.

En este plan se estableció como objetivo una calificación de 9.0 o más tres veces porque después de utilizar el simulador durante varios meses fue evidente que lograr esa calificación es difícil. Después de haber realizado el estudio inicial en donde los residentes y médicos tratantes realizaron artroscopías diagnósticas de rodilla, el promedio de calificación para todos los participantes fue de 6.96 en la artroscopía sin gancho palpador y 7.22 en la artroscopía con gancho palpador. En el programa de entrenamiento con el simulador de cirugía robótica Da Vinci, se estableció como meta de competencia para progresar a otras etapas una calificación de 7.5 en ejercicios de simulación. Esta calificación también discrimina entre niveles de habilidad quirúrgica. ${ }^{28}$

No se eligió un número de horas determinado de práctica, ya que diferentes personas pueden desarrollar una habilidad en un diferente período de tiempo. Se asume que si el usuario obtiene una calificación de nueve tres veces es suficientemente hábil para reproducir el resultado. Se eligió la calificación global como parámetro de evaluación, ya que ésta incluye casi todas las variables antes mencionadas y se considera que esta calificación se correlaciona con un adecuado nivel de competencia en la artroscopía.

La práctica en talleres con modelo en seco, talleres con modelos cadavéricos y permitir una mayor participación de los residentes en las cirugías es importante para mantener el interés de los residentes y permitir que progresen a escenarios más reales. También genera la percepción de que la práctica tiene una recompensa. 
Parte del objetivo de las evaluaciones semestrales en el simulador y en una cirugía real es correlacionar la práctica y la calificación en el simulador con el desempeño en el quirófano. Ésta es la parte crítica del plan de simulación. De esta manera se contará con datos objetivos para validar o no el plan de enseñanza con simulación. Así también se podrá proveer al residente con retroalimentación objetiva e identificar áreas de oportunidad en el desarrollo de habilidades motoras y cognitivas de los residentes.

Como limitaciones para llevar a cabo este plan de entrenamiento tenemos: la falta de simuladores en todas las sedes hospitalarias, así como carecemos en el país de la infraestructura para importarlos, almacenarlos y darles mantenimiento. También existe un problema de logística, ya que es poco probable que los residentes de los primeros dos años tengan tiempo libre destinado al desarrollo de habilidades motoras específicamente. Otra dificultad es la falta de disposición, apego al cambio y entrenamiento de los profesores de curso, de los médicos adscritos y de los mismos residentes para permitir la práctica y realizar una evaluación con base en su cumplimiento con el plan de entrenamiento. Finalmente, los residentes de menores grados académicos pueden mostrar más interés que los de mayores grados. Es importante mantener la anonimidad del residente y fomentar un ambiente seguro en donde se sientan tranquilos para que ellos mismos se interesen en el desarrollo de competencias a base de la práctica constante.

Es probable que se deban realizar ajustes al llevar a cabo el programa. Se debe realizar un plan piloto a corto plazo para evaluar la mejoría de las habilidades de los residentes y valorar si existe transferibilidad de habilidades. Los resultados globales del plan deberán evaluarse por lo menos con un par de generaciones de residentes que hayan sido sometidos al programa e, idealmente, compararlos con residentes que no han participado en el plan de entrenamiento.

Con las restricciones actuales en muchos programas de residencia y el interés creciente en simulación médica, es importante desarrollar planes de estudio estructurados y validados que incorporen la simulación para complementar el desarrollo de habilidades motoras en residentes de ortopedia.

Bibliografía

1. Dougherty PJ. CORR curriculum - orthopaedic education: Faculty development begins at home. Clin Orthop Relat Res. 2014; 472(12): 3637-43.

2. Rosen KR. The history of medical simulation. J Crit Care. 2008; 23(2): 157-66.

3. Hui Y, Safir O, Dubrowski A, Carnahan H. What skills should simulation training in arthroscopy teach residents? A focus on resident input. Int J Comput Assist Radiol Surg. 2013; 8(6): 945-53.

4. Akhtar K, Sugand K, Wijendra A, Standfield NJ, Cobb JP, Gupte CM. Training safer surgeons: How do patients view the role of simulation in orthopaedic training? Patient Saf Surg. 2015; 9: 11.

5. Kalraiya A, Buddhdev P. The TROJAN Project: creating a customized international orthopedic training program for junior doctors. Orthop Rev (Pavia). 2015; 7(1): 5750.

6. Pitts D, Rowley DI, Sher JL. Assessment of performance in orthopaedic training. J Bone Joint Surg Br. 2005; 87(9): 1187-91.
7. Coughlin RP, Pauyo T, Sutton JC 3rd, Coughlin LP, Bergeron SG. A validated orthopaedic surgical simulation model for training and evaluation of basic arthroscopic skills. J Bone Joint Surg Am. 2015; 97(17): 1465-71.

8. Bergfeld JA. Issues with accreditation and certification of orthopaedic surgery fellowships. J Bone Joint Surg Am. 1998; 80(12): 1833-6; discussion 1846-50.

9. Leonard M, Kennedy J, Kiely P, Murphy PG. Knee arthroscopy: how much training is necessary? A cross-sectional study. Eur J Orthop Surg Traumatol. 2007; 17(4): 359-62.

10. Van den Dobbelsteen JJ, Karahan M, Akgün U. Theory on psychomotor learning applied to arthroscopy. In: Karahan M, Kerkhoffs MMJG, Randelli P, Tuijthof JMG, editors. Effective training of arthroscopic skills. Berlin, Heidelberg: Springer Berlin Heidelberg; 2015. p. 17-32.

11. Hodgins JL, Veillette C. Arthroscopic proficiency: methods in evaluating competency. BMC Med Educ. 2013; 13: 61.

12. Stirling ER, Lewis TL, Ferran NA. Surgical skills simulation in trauma and orthopaedic training. J Orthop Surg Res. 2014; 9: 126.

13. Unalan PC, Akan K, Orhun H, Akgun U, Poyanli O, Baykan A, et al. A basic arthroscopy course based on motor skill training. Knee Surg Sports Traumatol Arthrosc. 2010; 18(10): 1395-9.

14. Gardner AK, Scott DJ, Pedowitz RA, Sweet RM, Feins RH, Deutsch ES, et al. Best practices across surgical specialties relating to simulation-based training. Surgery. 2015; 158(5): 1395-402.

15. Martin KD, Patterson DP, Cameron KL. Arthroscopic training courses improve trainee arthroscopy skills: a simulation-based prospective trial. Arthroscopy. 2016; 32(11): 2228-32.

16. Lubowitz JH, Provencher MT, Brand JC, Rossi MJ. Learning the language of Copernicus. Arthroscopy. 2015; 31(8): 1423-5.

17. Niitsu H, Hirabayashi N, Yoshimitsu M, Mimura T, Taomoto J, Sugiyama Y, et al. Using the objective structured assessment of technical skills (OSATS) global rating scale to evaluate the skills of surgical trainees in the operating room. Surg Today. 2013; 43(3): 271-5.

18. Hetaimish B, Elbadawi H, Ayeni OR. Evaluating simulation in training for arthroscopic knee surgery: a systematic review of the literature. Arthroscopy. 2016; 32(6): 1207-20.e1.

19. Garfjeld-Roberts P, Guyver P, Baldwin M, Akhtar K, Alvand A, Price AJ, et al. Validation of the updated ArthroS simulator: face and construct validity of a passive haptic virtual reality simulator with novel performance metrics. Knee Surg Sports Traumatol Arthrosc. 2017; 25(2): 616-25.

20. Slade-Shantz JA, Leiter JR, Gottschalk T, MacDonald PB. The internal validity of arthroscopic simulators and their effectiveness in arthroscopic education. Knee Surg Sports Traumatol Arthrosc. 2014; 22(1): 33-40.

21. Howells NR, Gill HS, Carr AJ, Price AJ, Rees JL. Transferring simulated arthroscopic skills to the operating theatre: a randomised blinded study. J Bone Joint Surg Br. 2008; 90(4): 494-9.

22. Boutefnouchet $\mathrm{T}$, Laios $\mathrm{T}$. Transfer of arthroscopic skills from computer simulation training to the operating theatre: a review of evidence from two randomised controlled studies. SICOT J. 2016; 2: 4.

23. Middleton RM, Baldwin MJ, Akhtar K, Alvand A, Rees JL. Which Global Rating Scale? A Comparison of the ASSET, BAKSSS, and IGARS for the Assessment of Simulated Arthroscopic Skills. J Bone Joint Surg Am. 2016; 98(1): 75-81.

24. Koehler RJ, Amsdell S, Arendt EA, Bisson LJ, Braman JP, Butler A, et al. The arthroscopic surgical skill evaluation tool (ASSET). Am J Sports Med. 2013; 41(6): 1229-37.

25. Hiemstra E, Kolkman W, Wolterbeek R, Trimbos B, Jansen FW. Value of an objective assessment tool in the operating room. Can J Surg. 2011; 54(2): 116-22.

26. Koehler RJ, Goldblatt JP, Maloney MD, Voloshin I, Nicandri GT. Assessing diagnostic arthroscopy performance in the operating room using the arthroscopic surgery skill evaluation tool (ASSET). Arthroscopy. 2015; 31(12): 2314-9.e2.

27. Bayona S, Akhtar K, Gupte C, Emery RJ, Dodds AL, Bello F. Assessing performance in shoulder arthroscopy: the imperial global arthroscopy rating scale (IGARS). J Bone Joint Surg Am. 2014; 96(13): e112.

28. Sridhar AN, Briggs TP, Kelly JD, Nathan S. Training in robotic surgery-an overview. Curr Urol Rep. 2017; 18(8): 58. 